\title{
Role of salt precursors for the synthesis of zinc oxide nanoparticles and in imparting variable antimicrobial activity
}

\author{
Manpreet Kaur ${ }^{*}$ and Anu Kalia ${ }^{2}$ \\ ${ }^{1}$ Department of Microbiology, Punjab Agricultural University, Ludhiana-141004 (Punjab), INDIA \\ ${ }^{2}$ Electron Microscopy and Nanoscience Laboratory, Punjab Agricultural University, Ludhiana-141004 (Punjab), \\ INDIA \\ *Corresponding author. E-mail: manpreet2126@gmail.com
}

Received: October 28, 2015; Revised received: March 4, 2016; Accepted: June 7, 2016

\begin{abstract}
Synthesis of nanoparticles (NPs) having unique potentials and properties is of great importance in nanotechnology. The NP synthesis techniques may include the wet chemistry to microbial incubation reduction methods. This work reports generation of ZnO NPs by identical preparation including incubation of different zinc salts i.e. zinc acetate, zinc chloride and zinc sulphate as precursors with cell free extracts of Bacillus circulans MTCC 7906 (Bc7906) and Pleurotus florida (Pf). The synthesized NPs exhibited variation in their absorption peaks in UV-Vis spectra which appeared at $275 \mathrm{~nm}, 325 \mathrm{~nm}$ and $375 \mathrm{~nm}$ with $P$. florida for the three salt precursors respectively while the Bc7906 generated ZnO NPs showed peaks between $300-350 \mathrm{~nm}$. A variation in ZnO NP morphology ranged from 50 to $120 \mathrm{~nm}$ in size and spherical, oval, cylindrical to trigonal anisotropic in shape by transmission EM. Further, the rough and corrugated surface topography of ZnO NPs was observed in Scanning EM. The \% weight for $\mathrm{Zn}$ element surface composition as recorded by SEM-EDS was observed to be highest for zinc acetate $(2.34 \%)$ and zinc sulphate $(7.54 \%)$ on microbial synthesis from Bc7906 and Pf respectively. The antimicrobial potential of the synthesized ZnO NPs on human pathogenic and plant beneficial bacteria was tested and it was observed to be highest for microbially synthesized ZnO NPs using zinc acetate (15 mm) and zinc sulphate $(14 \mathrm{~mm})$ as salt precursors @ 10 ppm. This is the first report on differential antimicrobial behavior of ZnO NPs on human pathogenic and plant beneficial microbes.
\end{abstract}

Keywords: Microscopy, Nanoparticles, UV-Vis spectroscopy, Zinc oxide

\section{INTRODUCTION}

The metal/ metal oxide NPs exhibit novel and improved physical, chemical and biological properties due to their larger surface area to volume ratios, nanoscale dimensions and random motion. These NPs may also exhibit potential antimicrobial properties particularly the ZnO NPs which hold considerable attention due to their unique catalytic antibacterial, antifungal and antiviral activities (Shrivastava et al., 2015). The NP synthesis protocols are as varied as the synthesized NPs spanning over physical, chemical and microbial methods. However, the microbial approach is most preferred due to involvement of less amount and types of chemicals and hence being more eco-friendly. The reports of synthesis of ZnO NPs using microbes like bacteria Aeromonas hydrophila (Jayaseelan et al., 2012), fungal filtrates of Aspergillus terreus (Baskar et al., 2013) and yeast Candida diversa strain JA1 (Chauhan et al., 2014) are known. The incubation with cell free or cell based extracts of microbes are especially recommended over higher eukaryotes as it renders ease of handling and easy genetic manipulation. The morphology and size of NPs can be controlled by altering the reaction time and by using different biotemplates (extracellular biomacromolecules from bacteria, fungi and yeast) and metal salts. The synthesis of spherical shaped ZnO NPs with size ranges between 20 $-40 \mathrm{~nm}$ using zinc acetate and spherobacterium Streptococcus thermophilus has been reported (Zhou et al., 2007). Similarly, another report on the use of probiotic bacteria Lactobacillus plantarum VITESO7 with zinc sulphate as metal salt precursor resulted in synthesis of spherical 7-17 nm ZnO NPs (Selvarajan and Mohanasrinivasan, 2013). Fungal filtrates of Alternaria alternata synthesized $\mathrm{ZnO}$ NPs of average size of $75 \mathrm{~nm}$ by using zinc sulphate solution (Sarkar et al., 2014). These NPs have a wide range of applications in pharmaceutics, paint and cosmetics industry. Moreover, $\mathrm{ZnO}$ is approved by FDA (Food and drug administration) to be used in sunscreens due to its ability to reflect the skin deteriorating UV radiation (Jin et al., 2009). The $\mathrm{ZnO}$ NPs have also been used in various textile, food and packaging industries owing to their antimicrobial properties probably because of production of reactive oxygen species on the surface of NPs $\mathrm{ZnO}$ possesses antibacterial activity both in microscale as well as nanoscale formulations (Nair et al., 2009). 
It also exhibits antisporicidal activity against high temperature and pressure resistant spores (Sawai, 2003). The concentrations may vary from $3 \mathrm{mmol} / \mathrm{l}$ to 12 $\mathrm{mmol} / \mathrm{l}$ or higher concentrations to partially and completely inhibit the spore germination (Liu et al., 2009). The antimicrobial activity of ZnO NPs is higher particularly against water-borne pathogens namely Shigella dysenteriae, Salmonella typhi, Vibrio cholera and Escherichia coli on doping with other semiconductor elements like cobalt (Oves et al., 2015). The ZnO NPs also possess antifungal activity against Aspergillus niger, A. fumigatus and A. aculeatus (Baskar et al., 2013). The present study aims to compare the use of different salt precursors and two microbial extracellular extracts for the synthesis of ZnO NPs. The antimicrobial activity of the synthesized NPs was then assessed against both pathogenic and soil beneficial microorganisms.

\section{MATERIALS AND METHODS}

Chemicals: Analytical grade zinc acetate dihydrate, zinc chloride and zinc sulphate of Hi-Media Laboratory chemicals Pvt. Ltd, Mumbai, India and the commercial zinc oxide nanoparticles were from Sisco Research Laboratory Pvt. Ltd., Mumbai, India were used. Microbial cultures: Microbial cultures, Bacillus circulans MTCC 7906, Escherichia coli, Pleurotus florida, Pseudomonas fluorescence, Rhizobium and Staphylococcus aurens were obtained from Department of Microbiology, Punjab Agricultural University, Ludhiana, Punjab, India. Enterobacter cloacae MTCC 509 was procured from IMTECH, Chandigarh, India.

Microbial synthesis of zinc oxide nanoparticles: The Bc7906 and Pf cultures were inoculated in nutrient broth and potato dextrose broth respectively and were incubated at $28^{\circ} \mathrm{C} \pm 2^{\circ} \mathrm{C}$ for 48 hours in BOD incubator at $120 \mathrm{rpm}$. For the synthesis of ZnO NPs, $200 \mathrm{ml}$ of cell free extract was incubated with $0.01 \mathrm{M}$ zinc acetate, zinc chloride and zinc sulphate respectively at $28^{0} \mathrm{C} \pm 2{ }^{0} \mathrm{C}$ for 24 hours in shaking and static conditions till complete conversion of zinc acetate, zinc chloride and zinc sulphate into ZnO NPs. Simultaneously, the culture (cell free extracts of Bacillus and Pleurotus alone) and chemical (zinc salt(s) in distill water) controls were also run along with the reaction flasks.

\section{Characterization of zinc oxide nanoparticles}

UV-Vis Spectroscopy: The samples were subjected to absorption spectroscopy with wavelength ranging from 200 to $800 \mathrm{~nm}$ using Elico SL 218 Double Beam UVVis Spectrophotometer. The absorbance was plotted against the wavelength to observe the characteristic peaks for synthesized nanoparticles.

Scanning electron microscopy (SEM) and Energy dispersive spectroscopy (EDS): Morphology of synthesized nanoparticles were characterized using SEM (Hitachi S-3400N) at $15 \mathrm{kV}$ accelerating voltage in secondary electron imaging mode. Sample was pre- pared by placing $10 \mu \mathrm{l}$ of the sample on the stub which was vacuum dried overnight and later sputter coated with gold Ion sputter coater (model Hitachi E-1010). The surface elemental composition in percentage of atom and weight present on the sample was analyzed by EDS (Thermo Noran) attached to SEM by using higher probe current (50 to 60 microamperes and aperture 2).

Transmission electron microscopy (TEM):The nanoparticle size and structure measurements were performed on TEM (Hitachi H-7650) at $80 \mathrm{kV}$ acceleration voltage in high contrast imaging mode. All the samples were sonicated for 20 minutes in a bath sonicator (Model- Equitron) to obtain even dispersion of the particles. Samples were drop casted by placing 10 $\mu l$ of the sample suspension on carbon/ formvar coated copper grid. The drop casted grids were air dried before imaging.

Antibacterial assay

Agar well assay (Modified Kirby-Bauer's method, 1966): Microbially synthesized nanoparticles were tested against three human pathogenic bacteria namely E. coli, E. cloacae MTCC 509 and S. aureus and three plant beneficial soil bacteria namely, Rhizobium sp., Azotobacter and Ps. fluorescens. Modified agar well diffusion method was used to check the antibacterial activity of the nanoparticles. Lawn cultures of the test microbes were prepared by spreading $100 \mu \mathrm{l}$ of freshly prepared log phase bacterial culture on the petriplates containing nutrient agar. Uniform wells of $0.5 \mathrm{~mm}$ diameter were made using a gel borer. Concentrations of 2, 5, 8 and $10 \mathrm{ppm}$ of the synthesized nanoparticles were added in these wells. Zinc acetate $(0.01 \mathrm{M})$, zinc chloride $(0.01 \mathrm{M})$, zinc sulphate $(0.01 \mathrm{M})$, bulk zinc oxide $(0.01 \mathrm{M})$, cell free extract of Bc7906 and Pf were used as control. The commercially available antibiotics were used as control i.e. Ampicillin, Chloroamphenicol, Penicillin G, Streptomycin, Sulphatriad and Tetracycline for Gram-positive microorganism and Ampicillin, Gentamicin, Nalidixic acid, Cefalexin and Co-Trimoxazol for Gram-negative microorganisms. The plates were incubated at $37{ }^{\circ} \mathrm{C}$ for 24 hours for human pathogenic cultures and at $25{ }^{\circ} \mathrm{C}$ for soil beneficial microorganism. After 24 hours, zone of inhibition ( $\mathrm{mm})$ were measured for each sample.

Viable cell count of cultures using dilution spread plate technique (Throndsen, 1978): All the bacterial cultures were grown in nutrient broth medium for 2448 hours under shaking conditions $(160 \mathrm{rpm}$ on a rotary shaker) for attaining log phase growth measured at $600 \mathrm{~nm}$ on spectrophotometer using filter 3. Three concentrations of NPs i.e. 5 ppm, 8 ppm and 10 ppm were mixed in $50 \mathrm{ml}$ nutrient broth in culture flasks and were ultra-sonicated for 15 minutes each. After sonication, $1 \mathrm{ml}$ of bacterial culture aliquot was inoculated in all the flasks and was mixed properly. Viable cell count on nutrient agar was enumerated at $0,2,4,6$ and 8 hours after application respectively. 
Statistical analysis: All the experiments were performed in triplicates. The mean values were calculated and reported as the mean \pm standard error represented in graphs. One way ANOVA using SPSS 20 software was conducted in agar well assay and two way ANOVA was done to determine the interaction among treatments and hours of incubation.

\section{RESULTS AND DISCUSSION}

UV-Vis spectroscopy: The microbially synthesized NPs under shaking as well as static conditions showed characteristic absorption maxima between 300-400 nm and 250-350 $\mathrm{nm}$ for Bc7906 and Pf extracts respectively (Figs. 1 and 2). As $\mathrm{ZnO}$ NPs generally have

Table 1. Antimicrobial activity of Zinc oxide nanoparticles microbially synthesized against human pathogenic and soil beneficial microorganisms.

\begin{tabular}{|c|c|c|c|c|c|c|c|c|}
\hline \multirow[b]{2}{*}{$\begin{array}{l}\text { Type of } \\
\text { synthesis }\end{array}$} & \multirow[b]{2}{*}{$\begin{array}{l}\text { Type of salt } \\
\text { precursor } \\
\text { used }\end{array}$} & \multirow[b]{2}{*}{$\begin{array}{l}\text { Conc. of } \\
\text { NPs used }\end{array}$} & \multicolumn{4}{|c|}{ Zone of inhibition (mm) } & \multirow[b]{2}{*}{$\begin{array}{l}\text { Ps. } \\
\text { fluorecens }\end{array}$} & \multirow[b]{2}{*}{ Azotobacter } \\
\hline & & & $\begin{array}{l}S . \\
\text { aureus }\end{array}$ & $\begin{array}{l}E . \\
\text { coli }\end{array}$ & $\begin{array}{l}\text { E. cloacae } \\
\text { MTCC } 509\end{array}$ & Rhizobium & & \\
\hline \multirow{12}{*}{$\begin{array}{l}\text { Bacterial } \\
\text { synthesis }\end{array}$} & \multirow[t]{4}{*}{ Zinc acetate } & $2 \mathrm{ppm}$ & 11 & - & - & - & - & - \\
\hline & & $5 \mathrm{ppm}$ & 11 & - & - & - & - & - \\
\hline & & $8 \mathrm{ppm}$ & 14 & - & 12 & - & - & 12 \\
\hline & & $10 \mathrm{ppm}$ & 15 & - & 14 & 11 & - & 13 \\
\hline & \multirow[t]{4}{*}{$\mathrm{ZnCl}_{2}$} & $2 \mathrm{ppm}$ & 9 & - & - & - & - & - \\
\hline & & $5 \mathrm{ppm}$ & 9 & - & - & - & - & - \\
\hline & & 8 ppm & 10 & - & - & - & - & - \\
\hline & & 10 ppm & 11 & - & 8 & 10 & - & - \\
\hline & \multirow{4}{*}{$\mathrm{ZnSO}_{4}$} & $2 \mathrm{ppm}$ & - & - & - & - & - & - \\
\hline & & $5 \mathrm{ppm}$ & - & - & - & - & - & - \\
\hline & & 8 ppm & 10 & - & - & - & - & - \\
\hline & & $10 \mathrm{ppm}$ & 14 & - & - & - & - & - \\
\hline \multirow{12}{*}{$\begin{array}{l}\text { Fungal syn- } \\
\text { thesis }\end{array}$} & \multirow[t]{4}{*}{ Zinc acetate } & $2 \mathrm{ppm}$ & - & - & - & - & - & - \\
\hline & & $5 \mathrm{ppm}$ & - & - & - & - & - & - \\
\hline & & 8 ppm & - & - & - & - & - & - \\
\hline & & 10 ppm & 13 & - & - & - & - & 12 \\
\hline & \multirow[t]{4}{*}{$\mathrm{ZnCl}_{2}$} & $2 \mathrm{ppm}$ & - & - & - & - & - & - \\
\hline & & $5 \mathrm{ppm}$ & - & - & - & - & - & - \\
\hline & & 8 ppm & - & - & - & - & - & - \\
\hline & & $10 \mathrm{ppm}$ & - & - & - & - & - & - \\
\hline & \multirow{4}{*}{$\mathrm{ZnSO}_{4}$} & $2 \mathrm{ppm}$ & - & - & - & - & - & - \\
\hline & & $5 \mathrm{ppm}$ & - & - & - & - & - & - \\
\hline & & 8 ppm & 10 & - & 10 & 9 & - & 18 \\
\hline & & 10 ppm & 13 & - & 12 & 10 & - & 21 \\
\hline \multirow{4}{*}{\multicolumn{2}{|c|}{ Commercial ZnO NP }} & $2 \mathrm{ppm}$ & 16 & 8 & 9 & 10 & 11 & 22 \\
\hline & & $5 \mathrm{ppm}$ & 20 & 9 & 11 & 14 & 12 & 24 \\
\hline & & 8 ppm & 22 & 9 & 13 & 15 & 13 & 25 \\
\hline & & $10 \mathrm{ppm}$ & 24 & 11 & 14 & 17 & 15 & 28 \\
\hline \multicolumn{2}{|c|}{ C.D @5\% } & & 2.06 & NS & 2.56 & 3.44 & NS & 4.34 \\
\hline
\end{tabular}

Table 2. Zone of inhibition against test microorganisms for specific antibiotics.

\begin{tabular}{lllllll}
\hline Antibiotics (concentration in mcg) & \multicolumn{7}{c}{ Zone of inhibition (mm) } \\
\cline { 2 - 6 } Hexa G-minus & S. aureus & $\begin{array}{l}\text { E. cloacae } \\
\text { MTCC 509 }\end{array}$ & E. coli & Rhizobium & Ps. fluorescens & Azotobacter \\
\hline Ampicillin (10 mcg) & - & - & - & - & 32 & 35 \\
Gentamicin (10 mcg) & 21 & 12 & 19 & 25 & 30 & 36 \\
Nalidixic acid (30 mcg) & - & 27 & - & - & 14 & 35 \\
Chloroamphenicol (30 mcg) & 24 & 26 & 30 & 12 & - & 32 \\
Cefalexin ( 30 mcg) & - & - & - & - & - & 35 \\
Co-Trimoxazole (25 mcg) & - & 20 & 25 & - & 11 & 36 \\
Hexa G-plus & - & - & - & 10 & - & 35 \\
Ampicillin (10 mcg) & - & - & - & - & - & 31 \\
Penicillin G (0.6 mcg) & - & - & - & - & 23 & 35 \\
Streptomycin (10 mcg) & - & - & 14 & 22 & 12 & 36 \\
Sulphatriad (30 mcg) & 20 & 23 & 29 & 33 & 17 & NS \\
Tetracycline (25 mcg) & 2.31 & 2.91 & 2.90 & 3.70 & 2.89 & \\
C.D @ 5 \% & & & & &
\end{tabular}


Table 3. Antimicrobial activity of chemical controls used against pathogenic microorganisms and soil beneficial microorganisms.

\begin{tabular}{|c|c|c|c|}
\hline \multirow[b]{2}{*}{ Controls } & \multirow[b]{2}{*}{ E.coli } & \multicolumn{2}{|l|}{ Zone of inhibition (mm) } \\
\hline & & E. cloacae MTCC 509 & Ps. fluorescens \\
\hline ZnO Bulk 2ppm & - & - & - \\
\hline $5 \mathrm{ppm}$ & - & - & - \\
\hline $8 \mathrm{ppm}$ & - & - & - \\
\hline $10 \mathrm{ppm}$ & - & - & - \\
\hline $\mathrm{ZnCl}_{2} 2 \mathrm{ppm}$ & - & - & - \\
\hline $5 \mathrm{ppm}$ & - & - & - \\
\hline $8 \mathrm{ppm}$ & - & - & - \\
\hline $10 \mathrm{ppm}$ & - & - & - \\
\hline Zinc acetate 2 ppm & - & 10 & - \\
\hline $5 \mathrm{ppm}$ & - & 11 & 14 \\
\hline 8 ppm & 13 & 15 & 14 \\
\hline $10 \mathrm{ppm}$ & 13 & 15 & 14 \\
\hline $\mathrm{ZnSO}_{4} 2 \mathrm{ppm}$ & - & - & - \\
\hline $5 \mathrm{ppm}$ & - & - & - \\
\hline $8 \mathrm{ppm}$ & 22 & - & - \\
\hline $10 \mathrm{ppm}$ & 22 & - & - \\
\hline C.D @ 5\% & 2.31 & 2.31 & NS \\
\hline
\end{tabular}

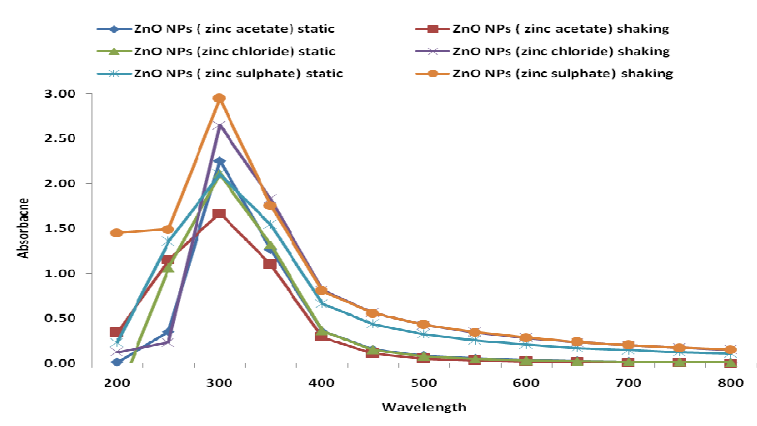

Fig. 1. UV-vis spectra of ZnO NPs synthesized from cell free extract of Bc7906.

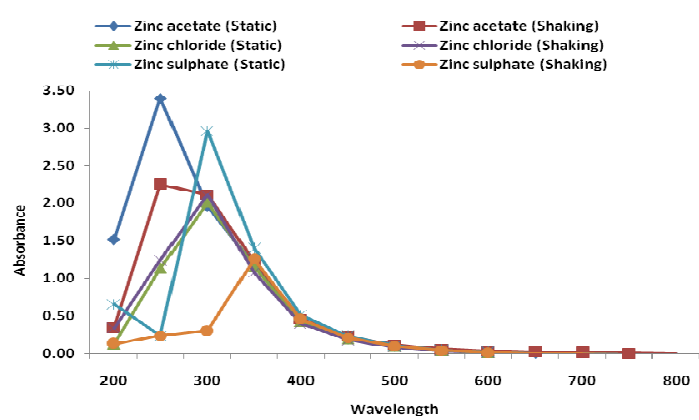

Fig. 2. UV-vis spectra of $\mathrm{ZnO} N P$ s synthesized from cell free extract of $P f$.

peaks ranging from 200-300 nm wavelength (Kumar and Rani, 2013), the absortion maxima for our NPs have shown a red shift. Similar peaks at about $340 \mathrm{~nm}$ and $374 \mathrm{~nm}$ have been reported for A. terrus (Baskar et al., 2013) and A. hydrophila synthesized ZnO NPs (Jayaseelan et al., 2012).

Scanning electron microscopy (SEM): The ZnO NPs obtained by keeping microbial cell extracts with precursor salts under shaking conditions showed uniform size and shapes. Hence, these NPs were furthered used for the later characterization and antimicrobial activity tests. The SEM studies revealed synthesis of small size $\mathrm{ZnO}$ NPs existing in both homogenously monodis-

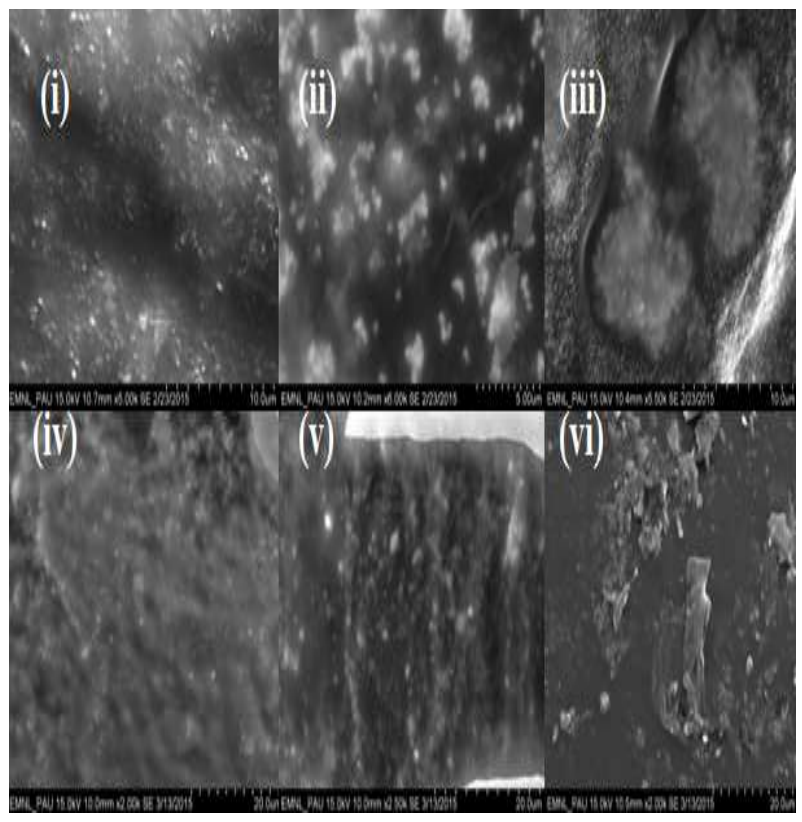

Fig. 3. SEM images of $\mathrm{ZnO}$ nanoparticles synthesized from cell free extract of Bc7906 using (i) zinc acetate (ii) zinc chloride (iii) zinc sulphate as salt precursors. ZnO nanoparticles synthesized from cell free extract of Pf using (iv) Zinc acetate $(v)$ zinc chloride (vi) zinc sulphate as salt precursors.

persed as well as in aggregated form (Fig.3). The SEM -EDS analysis of the different ZnO NPs in X-ray spectrum mode showed characteristic peaks for Zinc (L $($ alpha $)=1.012 \mathrm{keV}, \mathrm{K} \alpha=8.630 \mathrm{keV}$ and $\mathrm{K} \beta=9.570$ $\mathrm{keV})$. It also gave \% atom and \% weight for the presence of zinc element which was highest $(2.34 \%)$ in zinc acetate precursor derived $\mathrm{ZnO}$ NPs as compared to other two salt precursors incubated with Bc7906 culture (Fig.4). While the Pf derived ZnO NPs from zinc sulphate as precursor had highest percent weight for $\mathrm{Zn}$ element $(7.54 \%)$ on their surface. Similar observation of signal peak for the presence of $\mathrm{Zn}$ element on the surface of $\mathrm{ZnO}$ NPs has been reported by Jayaseelan et al. (2012). 

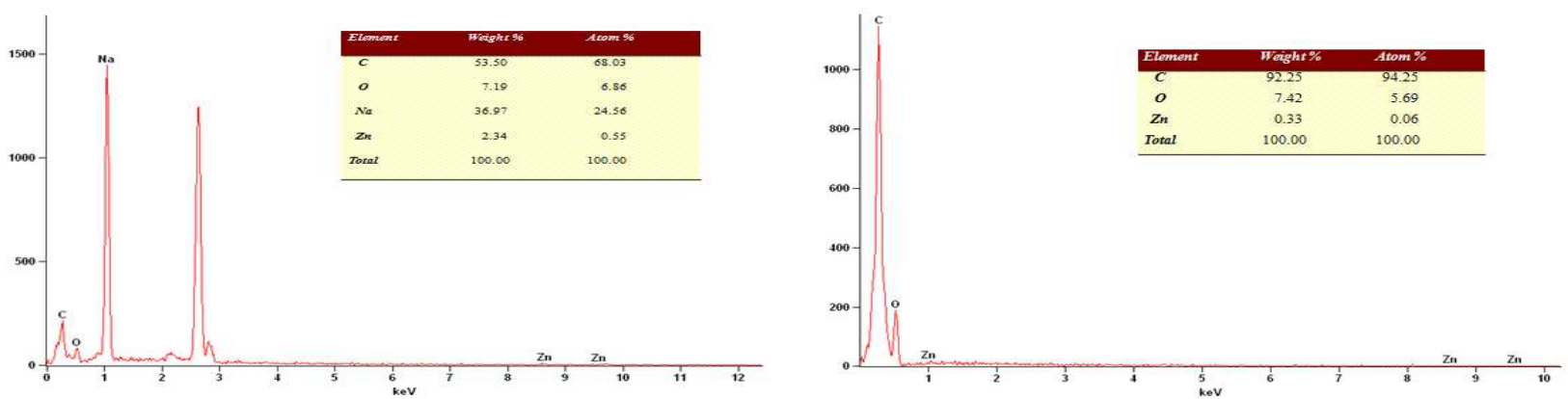

a) zinc acetate

d) zinc acetate
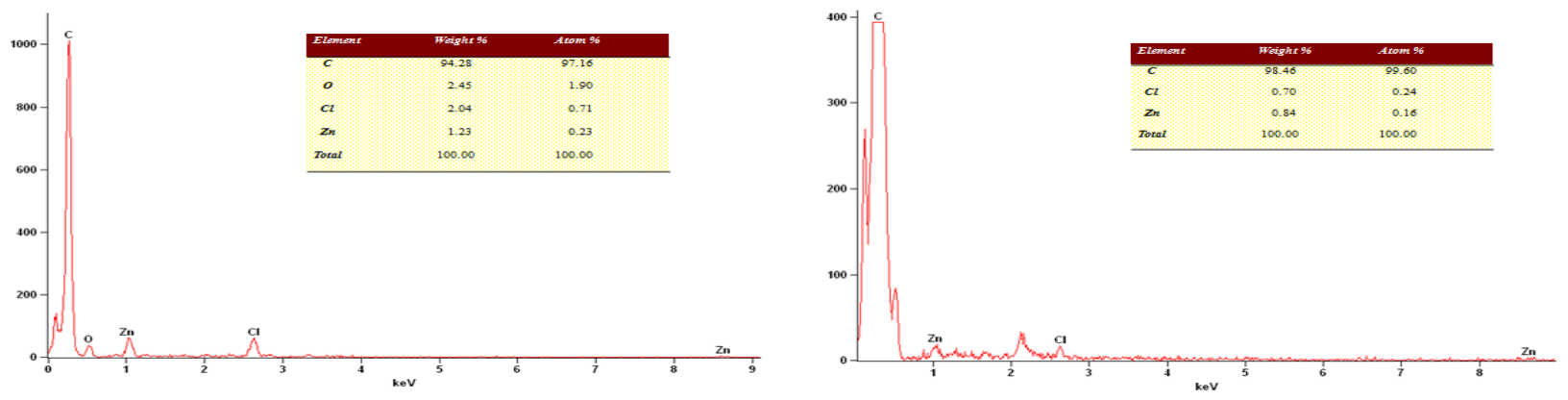

b) zinc chloride

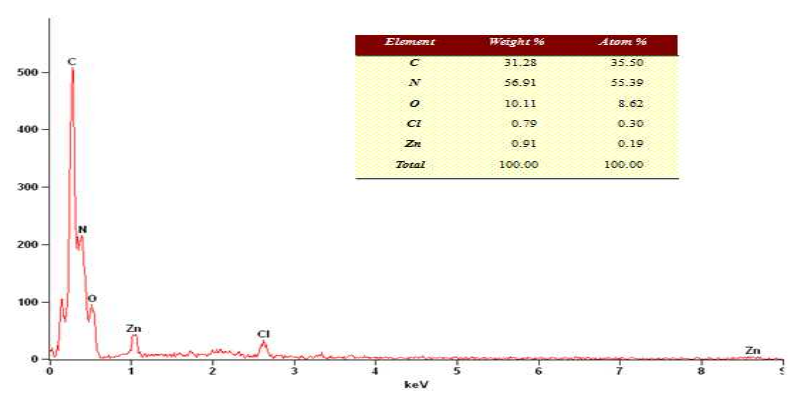

e) zinc chloride

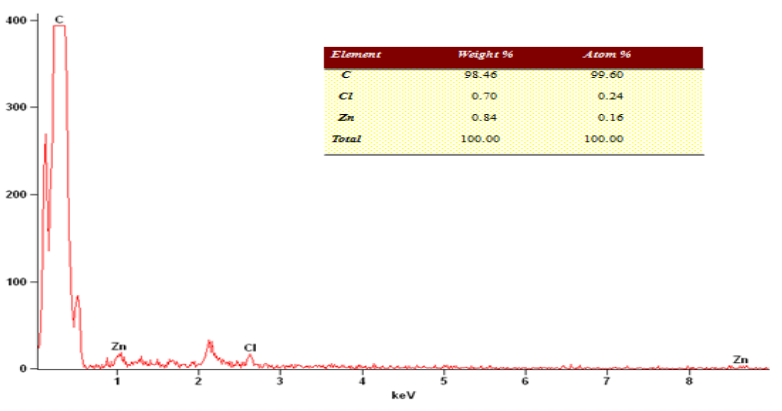

c) zinc sulphate

f) zinc sulphate

Fig. 4. SEM-EDS spectral peaks of ZnO NPs synthesized from cell free extract of Bc7906 using (a) zinc acetate (b) zinc chloride (c) zinc sulphate as salt precursors. ZnO nanoparticles synthesized from cell free extract of Pf using (d) Zinc acetate (e) zinc chloride $(f)$ zinc sulphate as salt precursors.

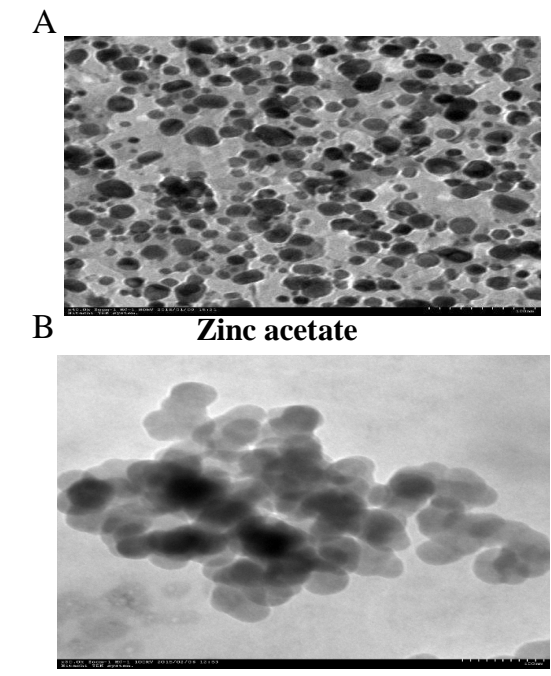

a) Zinc acetate
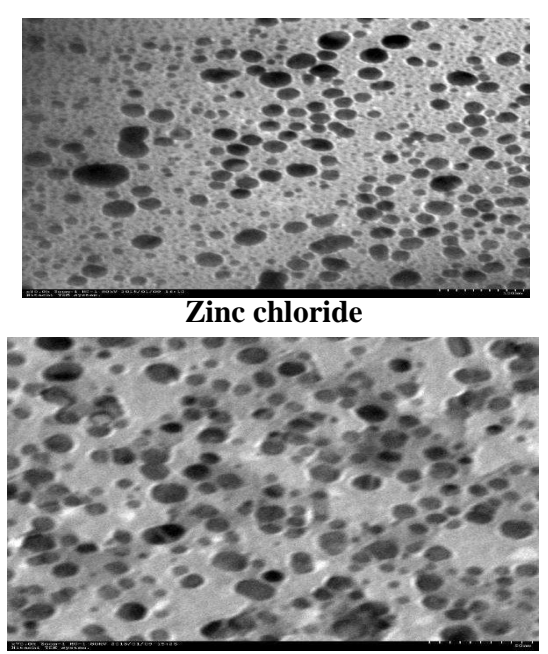

b) Zinc chloride
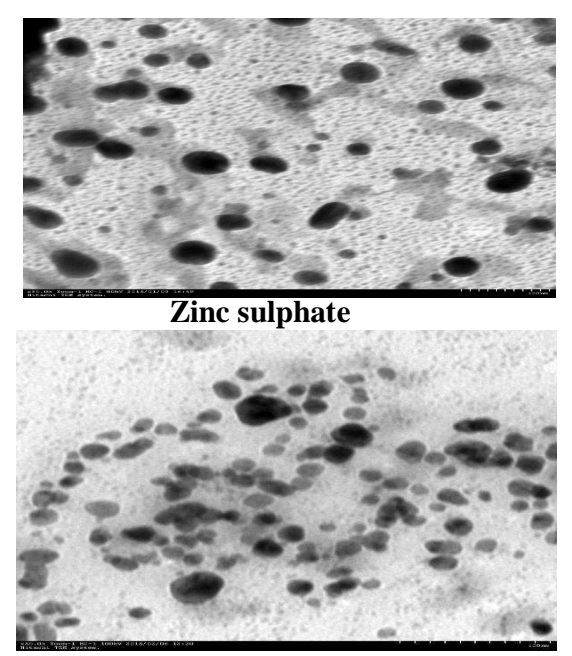

c) Zinc sulphate

Fig.5. Transmission electron micrographs of zinc oxide nanoparticles A) synthesized from Bc7906 and B) Pf 
A

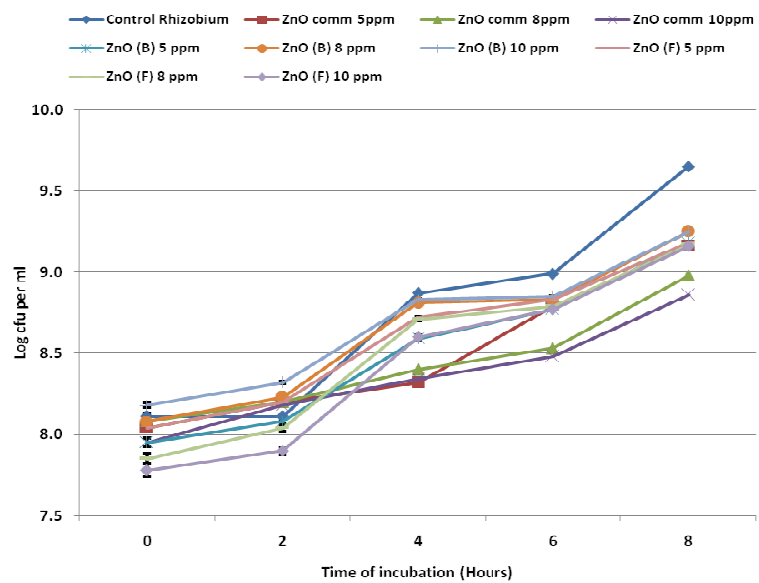

B

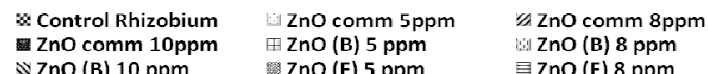

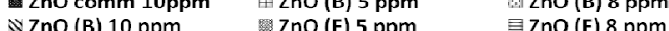

(B) $\mathrm{ZnO}$ (B) $10 \mathrm{ppm}$

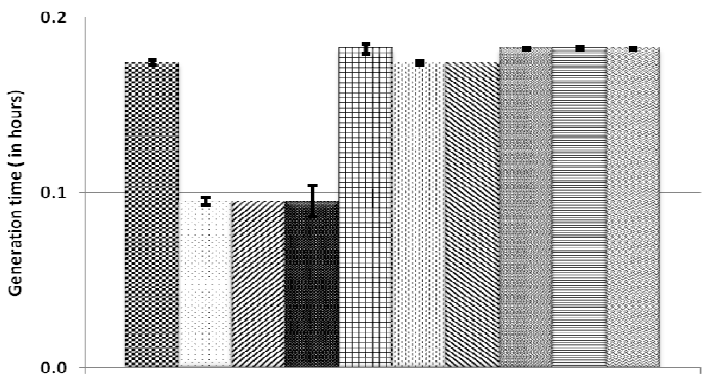

Time of incubation (4th hour)

C.D @ $5 \%$ A $($ Hours of incubation $)=0.017 \mathrm{~B}($ Treatments $)=0.024 \mathrm{~A} \times \mathrm{B}=0.053$

Fig. 6. Growth measurements for Rhizobium treated with zinc oxide nanoparticles A) Growth curve (Log cfu per ml) of Rhizobium cultures B) Generation time growth curve against hours of incubation.

A

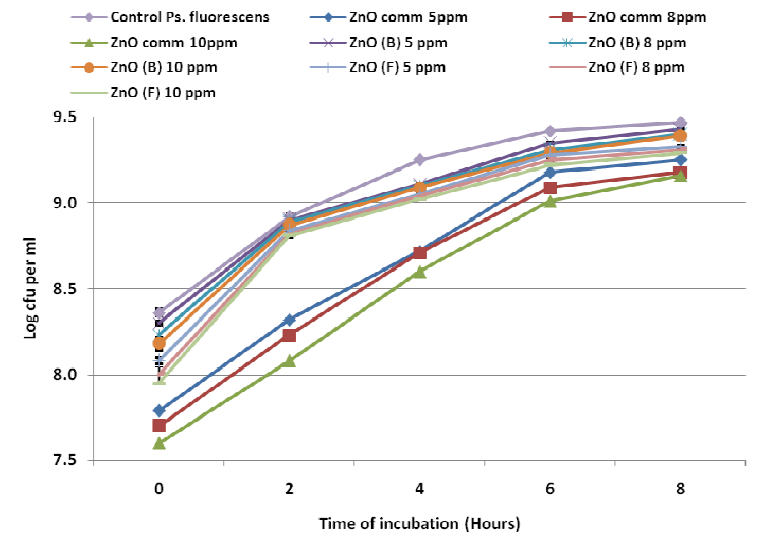

B
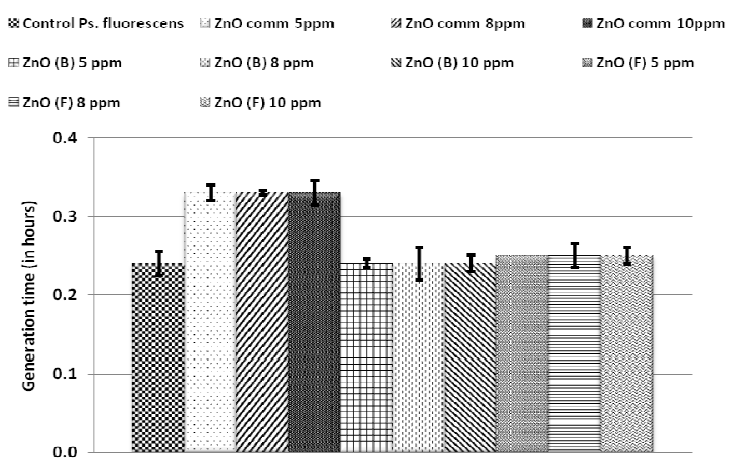

Time of incubation (8th hour)

C.D @ $5 \%$ A (Hours of incubation $)=0.019 \mathrm{~B}$ (Treatments $)=0.027 \mathrm{~A} \times \mathrm{B}=0.06$

Fig. 7. Growth measurements for Ps. fluorescens treated with zinc oxide nanoparticles A) Growth curve (Log cfu per ml) of Ps. fluorescens cultures B) Generation time growth curve against hours of incubation.

A

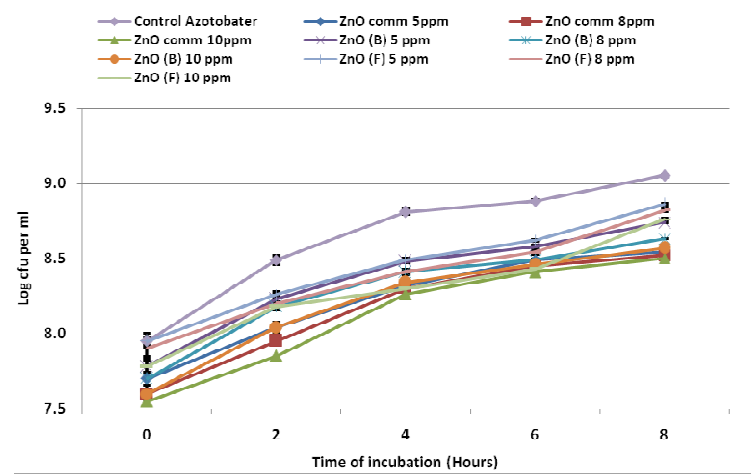

B

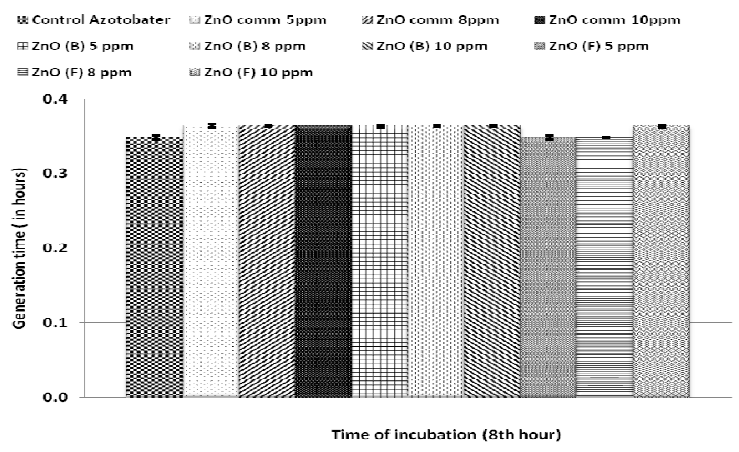

C.D @ $5 \%$ A $($ Hours of incubation $)=0.0241 \mathrm{~B}($ Treatments $)=0.0341 \mathrm{~A} \times \mathrm{B}=0.076$

Fig. 8. Growth measurements for Azotobacter treated with zinc oxide nanoparticles A) Growth curve (Log cfu per ml) of Azotobacter cultures B) Generation time growth curve against hours of incubation. 
A

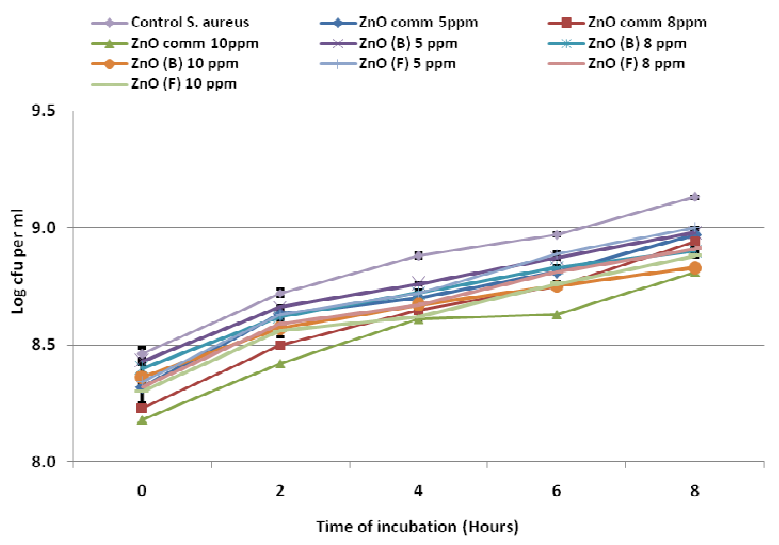

B

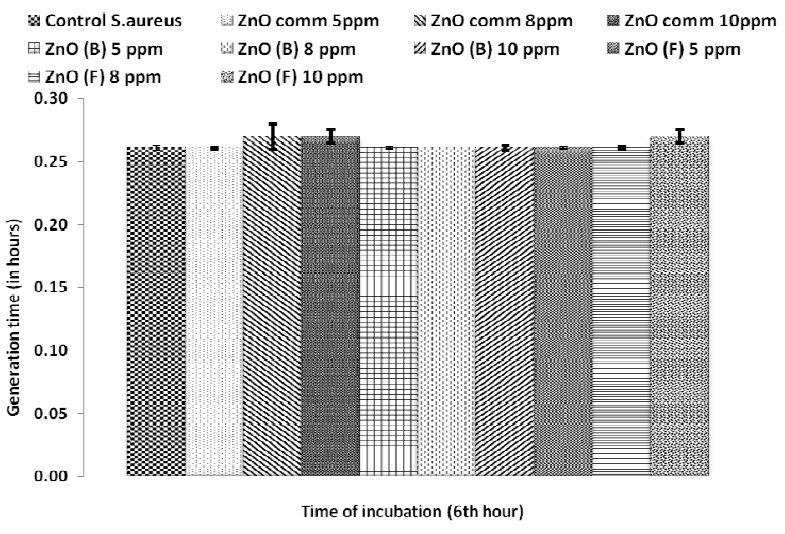

C.D @ $5 \%$ A (Hours of incubation $)=0.016$ B (Treatments $)=0.023 \mathrm{~A} \times \mathrm{B}=0.051$

Fig. 9. Growth measurements for S. aureus treated with zinc oxide nanoparticles A) Growth curve (Log cfu per ml) of staphylococcal cultures B) Generation time against hours of incubation.

A

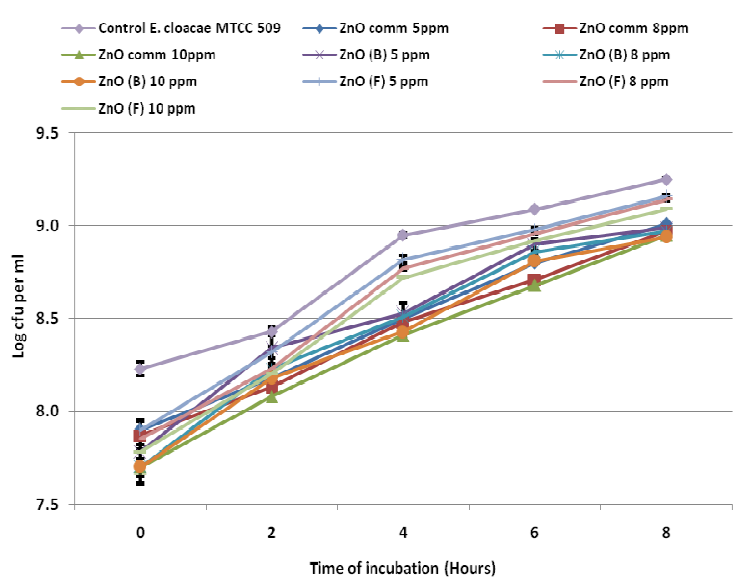

B

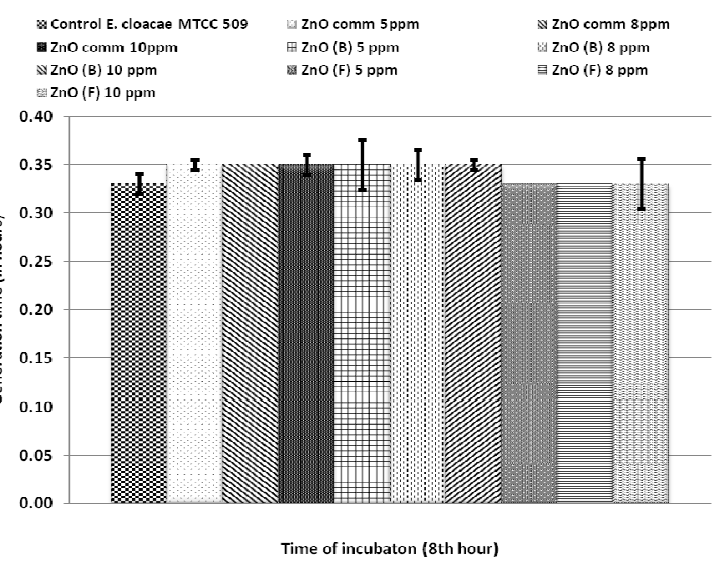

C.D @ $5 \%$ A (Hours of incubation $)=0.034 \mathrm{~B}($ Treatments $)=0.047 \mathrm{~A} \times \mathrm{B}=0.106$

Fig. 10. Growth measurements for E. cloacae MTCC 509 treated with zinc oxide nanoparticles A) Growth curve (Log cfu per ml) of E. cloacae MTCC 509 B) Generation time against hours of incubation.

A

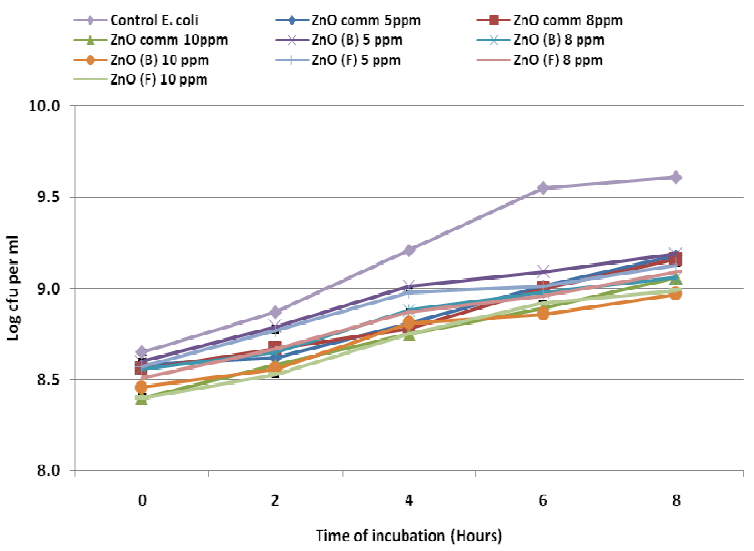

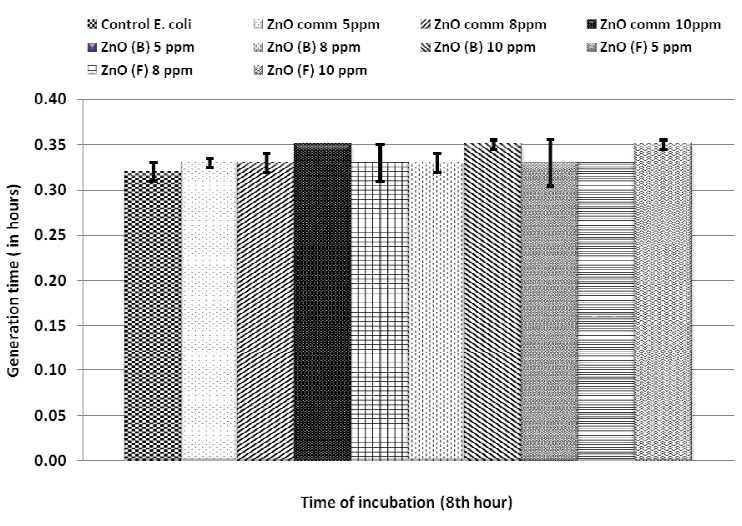

C.D @ $5 \%$ A (Hours of incubation $)=0.031$ B (Treatments $)=0.044 \mathrm{~A} \times \mathrm{B}=0.098$

Fig. 11. Growth measurements for E. coli treated with zinc oxide nanoparticles A) Growth curve (Log cfu per ml) of E. coli B) Generation time against hours of incubation. 
Transmission electron microscopy (TEM): The reduction of all the three precursor zinc salts with the microbial cell free extracts under shaking condition generated oval to nearly spherical shaped NPs. However, the Pf extracts had the smallest size NPs (10-20 nm) synthesized using zinc sulphate precursor (Fig.5). Other precursors on incubation with the microbial cultures also yielded spherical shaped NPs. Thus the type of salt precursor has negligible effect on the morphology of the microbial synthesized ZnO NPs unlike the wet chemistry synthesis where altered morphologies were obtained on basis of precursor salt used (Mayekar et al., 2014). Similar synthesis of spherical shaped NPs with size range between $30-63 \mathrm{~nm}$ has been reported by Meruvu et al. (2011). Kundu et al. (2014) have also reported synthesis of 100-200 nm ZnO NPs by actinobacteria Rhodococcus pyridinivorans NT2 in presence of $\mathrm{ZnSO}_{4}$ salt.

Agar well assay: The commercial ZnO NPs @ 10 ppm showed significantly higher antimicrobial activity (AMA) against all the test microorganisms. They showed maximum AMA against gram positive $S$. aureus with $24 \mathrm{~mm}$ followed by $14 \mathrm{~mm}$ zone of inhibition against E. cloacae MTCC 509. Lesser inhibition was observed against other gram negative pathogenic cultures like $11 \mathrm{~mm}$ only for E. coli. A higher AMA was observed as zone of inhibition against plant beneficial microorganism with highest against Azotobacter $(28 \mathrm{~mm})$ and lowest against $P$ s. fluorescens $(15 \mathrm{~mm})$ followed by Rhizobium $(18 \mathrm{~mm})$. Zinc acetate derived Bc7906 incubated ZnO NPs formed under shaking conditions showed maximum AMA @ 10 ppm concentration against $E$. cloacae MTCC $509(17 \mathrm{~mm})$ followed by $S$. aureus with $15 \mathrm{~mm}$ zone of inhibition (Table 1). Similar report of AMA of ZnO NPs at a higher concentration of $100 \mu \mathrm{g} / \mathrm{ml}$ against $S$. aureus with $14.52 \mathrm{~mm}$ zone of inhibition has been documented by Vani et al. (2011). Interestingly, these NPs exhibited lesser inhibition against gram negative plant beneficial rhizobacteria Rhizobium and Azotobacter, while no inhibition was observed against enterobacteriaceae member human pathogenic E. coli and plant probiotic pseudomonadaceae family member $P$ s. fluorescens. Contrary to our results, $\mathrm{ZnO}$ NPs synthesized from A. hydrophila have been reported to inhibit $E$. coli (Jayaseelan et al., 2012) as well as had shown highest AMA against Ps. aeruginosa. The AMA of $\mathrm{ZnO}$ NPs synthesized from Pf using zinc sulphate as precursor showed maximum zone of clearance of 21 $\mathrm{mm}$ against plant beneficial gram negative Azotobacter and $17 \mathrm{~mm}$ each against gram positive human pathogenic $S$. aureus and gram negative $E$. cloacae MTCC 509. These NPs had smaller inhibition zones against Ps. fluorescens and Rhizobium and exhibited no inhibition against $E$. coli. Hence, $E$. coli seems to be resistant against both types of microbially synthesized $\mathrm{ZnO}$ NPs. This may probably due to presence of some capping protein(s) non-homologous to E. coli cell surface ligands or other proteins. The chemical controls (Bulk $\mathrm{ZnO}$ and Zinc chloride) showed no inhibitory effect on any of the test pathogens and soil beneficial microorganisms. Thus it could be ruled out that the lower concentrations of the ionic zinc $\left(\mathrm{Zn}^{2+}\right)$ released by these compounds were lethal. Chemical control (Zinc acetate) exhibited slight inhibitory activity against $E$. coli, E. cloacae MTCC 509 and Ps. fluorescens while zinc sulphate inhibited the growth of $E$. coli. Therefore, the chemical composition of the zinc salt utilized affects the AMA it renders as zinc acetate and zinc sulphate exhibited some inhibitory effect on the microbial growth. Some antibiotics showed better inhibition than the NPs such as Nalidixic acid and Chloroamphenicol with maximum zone of inhibition against $E$. cloacae MTCC 509. However, the concentrations being used were more in comparison to the nanoparticle concentrations used in this report. Strikingly, Cefalexin and ampicillin does not showed any inhibition against any of the pathogenic microorganisms. Diameter of zone of inhibitions by antibiotics against E. cloacae MTCC 509, E. coli, S. aureus, Rhizobium, Ps. fluorescens and Azotobacter are presented in Table 3. Taking in account the size of the inhibition zone formed by these NPs, commercial ZnO NPs @ 10 ppm are more effective in terms of their potential as antimicrobial agents compared to microbially synthesized ZnO NPs at the same concentration probably due to faster ionization yielding $\mathrm{Zn}^{2+}$ ions leading to higher toxicity and eventually delay in cell growth followed by death.

Growth of microbial cultures in broth supplemented with NPs. Various ZnO NPs synthesized using different salt precursors showed significant difference among their antimicrobial activity. Zinc acetate derived $\mathrm{ZnO}$ NPs generated from the cell free extract of Bc7906 and zinc sulphate derived $\mathrm{ZnO}$ NPs generated from cell free extract of Pf under shaking conditions were selected further for broth-NPs interaction study to determine the extent of cell morphological alteration on incubation with $\mathrm{ZnO}$ NPs as well to determine the variation in the generation time of the test microbes. The growth of Rhizobium in the presence of different $\mathrm{ZnO}$ NP concentration varied significantly. The rhizobial growth in control treatment without NP supplementation increased with the increase in hours of incubation. Rhizobium culture treated with commercial $\mathrm{ZnO}$ NPs exhibited a concentration dependent decrease i.e. lowest log cfu per ml @ 10 ppm followed by $8 \mathrm{ppm}$ and $5 \mathrm{ppm}$ concentrations. The zinc sulphate derived $\mathrm{ZnO}$ NPs using Pf cell free extract @ 10 ppm showed the lower log cfu per $\mathrm{ml}$ as compared to other microbially synthesized ZnO NPs (Fig.6). Other NP inoculated cultures showed significantly lesser growth in broth media than the control Rhizobium culture. However, in all the $\mathrm{ZnO}$ treatments, growth was retarded with increase in concentration of NPs. Generation time was observed to be highest by the Rhizobium culture inoculated with fungal derived $\mathrm{ZnO}$ NPs using 
zinc sulphate @ 5,8 and 10 ppm as compared to control as well as other treatments. In the absence of any NPs, Ps. fluorescens growth increased with increase in hours of incubation (Fig. 7). Incubation of Ps. fluorescens cells in the presence of all the ZnO NPs synthesized using different salt precursors enhanced their growth in a time dependent manner with growth equivalent to control culture treatment. Thus, $\mathrm{ZnO}$ NPs even at higher concentration (10 ppm) have enhancing effect on Pseudomonas growth. But contrary to that, commercial ZnO NPs @ 5, 8 and 10 ppm have retarded the growth of Ps. fluorescens with lowest log cfu per ml. The generation time determined after 8 hours of incubation showed variation among the treatments giving the evidence for results reported by the enumeration of viable cell count. The results indicated that the highest generation time was reported by commercial ZnO NPs @ 5,8 and 10 ppm treated Ps. fluorescens cultures. Similarly, growth of Azotobacter treated and untreated with NPs increased with time (Fig. 8). However, on incubation with commercial ZnO NPs@10 ppm maximum growth was curbed compared to other treatments. This may be attributed to higher rate of dissolution and formation of $\mathrm{Zn}^{2+}$ ions and hence greater toxicity of commercial ZnO NPs. The growth reported by the determination of generation time after 8 hours of incubation also showed the greater toxicity of commercial ZnO NPs @ 10 ppm giving the highest generation time as compared to control culture of Azotobacter. $\mathrm{ZnO}$ NPs have an inhibitory effect on $S$. aureus growth. Both microbially synthesized and commercial ZnO NPs at 10 ppm concentration have retarded the bacterial growth in comparison to other lower concentrations used. These treatments@10 ppm were also reported to give highest generation time confirming the above stated results of growth inhibition.

As depicted in Fig. 10, a significant decrease in growth of the control and ZnO NPs treated E. cloacae MTCC 509 culture was observed. Highest growth inhibition was observed by both microbially generated $\mathrm{ZnO}$ NPs and commercial ZnO NPs @ 10 ppm giving lowest log cfu per $\mathrm{ml}$ and as well as highest generation time after 8 hours of incubation in comparison to control treatment. The decrease in growth exhibited NP concentration dependence. Chitra et al. (2013) have reported similar decrease in growth of $E$. coli and $P$. aeruginosa with increase in concentration of $\mathrm{ZnO}$ nanoparticles and maximum inhibition was obtained at $100 \mu \mathrm{g}$ per $\mathrm{ml}$ concentration. The $\log$ cfu per $\mathrm{ml}$ of $E$. coli cells in presence of $\mathrm{ZnO}$ NPs was decreased with increasing concentration of NPs (Fig.11). However, this is not in consensus to the formation of no zone of inhibition in the agar well study. This signifies the higher potential of same concentration of ZnO NPs under aqueous suspensions over agar gel embedded NPs. Here in this case, lowest log cfu per $\mathrm{ml}$ and highest generation time was reported by the $E$. coli culture treated with $\mathrm{ZnO}$ NPs synthesized from Bc7906 and commercial $\mathrm{ZnO}$
NPs@ 10 ppm in comparison to control culture after 8 hours of incubation. Similar report of inhibition of $E$. coli by $\mathrm{ZnO}$ NPs in broth at $15 \mathrm{mM}$ concentration has been documented by Paul and Ban (2014). Likewise, inhibition of growth of $E$. coli $\mathrm{O} 157: \mathrm{H} 7$ by application of ZnO NPs (@ 3 mmol/l) and @ 12 mmol/l or higher concentrations to completely inhibit the growth have been reported by Liu et al. (2009).

\section{Conclusion}

The microbially synthesized ZnO NPs had spherical morphologies with size ranging from 50 to $100 \mathrm{~nm}$. Both bacterial and fungal derived ZnO NPs showed antimicrobial activity against the test human pathogenic and plant beneficial microorganisms. The commercial ZnO NPs exhibited higher bacterial cytotoxicity showing $28 \mathrm{~mm}$ zone of inhibition@10 ppm against Azotobacter (a plant beneficial microorganism) as compared to microbially synthesized NPs. Hence, this differential toxicity of microbially generated $\mathrm{ZnO}$ NPs can be better utilized for developing novel agri-food commercial products keeping in view their lesser post application ecotoxicity issues observed as lesser or rather positive effect on plant beneficial rhizobacteria.

\section{ACKNOWLEDGEMENTS}

The authors are sincerely thankful to Dr. (Mrs.) Veena Khanna, Dr. G.S. Kocher, Dr. Madhurama Gangwar and Dr. H.S. Sodhi from Department of Microbiology, PAU, Ludhiana for providing the microbial cultures.

\section{REFERENCES}

Baskar, G., Chandhuru, J., Fahad, K.S. and Praveen, A.S. (2013). Mycological synthesis, characterization and antifungal activity of zinc oxide nanoparticles. Asian Journal of Pharmaceutical Science and Technology, 3 (4): 142-146.

Bauer, A.W., Kirby, W.M.M., Sherris, J.C. and Truck, M. (1966). Antibiotic susceptibility testing by standardized single disk method. American Journal of Clinical Pathology, 36: 493-496.

Chauhan, R., Reddy, A. and Abraham, J. (2014). Biosynthesis and antimicrobial potential of silver and zinc oxide nanoparticles using Candida diversa strain JA1. Der Pharma Chemica, 6(3):39-47.

Chitra, K. and Annadurai, G. (2013). Antimicrobial activity of wet chemically engineered spherical shaped $\mathrm{ZnO}$ nanoparticles on food borne pathogen. International Food Research Journal, 20:59-64.

Jayaseelan, C., Rahumana, A.A., Kirthi, A.V., Marimuthua, S., Santhoshkumara, T., Bagavana, A., Gaurav, K., Karthik, L. and Bhaskara Rao, K.V. (2012). Novel microbial route to synthesize $\mathrm{ZnO}$ nanoparticles using Aeromonas hydrophila and their activity against pathogenic bacteria and fungi. Spectrochimica Acta Part A, 90: 78- 84

Jin, T., Sun, D., Su, J.Y., Zhang, H. and Sue, H.J. (2009). Antimicrobial efficacy of zinc oxide quantum dots against Listeria monocytogenes, Salmonella enteritidis 
and Escherichia coli O157:H7. Journal of Food Science, 74(1): 46-52.

Kumar, H. and Rani, R. (2013). Structural and optical characterization of $\mathrm{ZnO}$ nanoparticles synthesized by microemulsion route. International Letters of Chemistry, Physics and Astronomy, 14: 26-36.

Kundu, D., Hazra, C., Chatterjee, A., Chaudhari, A. and Mishra, S. (2014). Extracellular biosynthesis of zinc oxide nanoparticles using Rhodococcus pyridinivorans NT2: Multifunctional textile finishing, biosafety evaluation and in vitro drug delivery in colon carcinoma.

Journal of Photochemistry and Photobiology B: Biology, 140: 194-204.

Liu, Y., He, L., Mustapha, A., Li, H. and Hu, Z.Q. (2009). Antibacterial activities of zinc oxide nanoparticles against Escherichia coli O157:H7. Journal of Applied Microbiology, 107: 1193-1201.

Mayekar, J., Dhar, V. and Radha, S. (2014). Role of salt precursor in the synthesis of zinc oxide nanoparticles. International Journal of Research in Engineering and Technology, 3 (3): 43-45.

Meruvu, H., Vangalapati, M., Chippada, S.C. and Bammidi, S.R. (2011). Synthesis and characterization of zinc oxide nanoparticles and its antimicrobial activity against Bacillus subtilis and Escherichia coli. Rasayan Journal of Chemical, 4 (1):217-222.

Nair, S., Sasidharan, A., Divya Rani, V.V., Menon, D., Nair, S., Manzoor, K. and Raina, S. (2009). Role of size scale of $\mathrm{ZnO}$ nanoparticles and microparticles on toxicity toward bacteria and osteoblast cancer cells. Journal of Materials Science-Materials in Medicine, 1:235-241.

Oves, M., Arshad, M., Khan, M.S., Ahmed, A.S., Azam, A. and Ismail, I.M.I. (2015). Anti-microbial activity of cobalt doped zinc oxide nanoparticles: Targeting water borne bacteria. Journal of Saudi Chemical Society, 19 (5): 581-588.

Paul, S. and Ban, D.K. (2014). Synthesis, characterization and the application of zinc oxide nanoparticles in biotechnology. International Journal of Advances in Chemical Engineering and Biological Sciences, 1 (1): 1-5.

Sarkar, J., Ghosh, M., Mukherjee, A., Chattopadhyay, D. and Acharya, K. (2014). Biosynthesis and safety evaluation of $\mathrm{ZnO}$ nanoparticles. Bioprocess and Biosystems Engineering, 37: 165-171.

Sawai, J. (2003). Quantitative evaluation of antibacterial activities of metallic oxide powders $(\mathrm{ZnO}, \mathrm{MgO}$ and $\mathrm{CaO}$ ) by conductimetric assay. Journal of Microbiological Methods, 54: 177-182.

Selvarajan, E. and Mohanasrinivasan, V. (2013). Biosynthesis and characterization of $\mathrm{ZnO}$ nanoparticles using Lactobacillus plantarum VITESO. Mat. Lett., 112: 180-182.

Shrivastava, V., Chauhan, P.S. and Tomar, R.S. (2015). Nanobiotechnology: A potential tool for biomedics. World Journal of Pharmacy and Pharmaceutical Sciences, 4 (5): 1929-1943.

Throndsen, J. (1978). The dilution culture method. In: Sournia A (ed) Phytoplankton manual Pp: 218-224. Unesco, Paris.

Vani, C., Sergin, G.K. and Annamalai, A. (2011). A study on the effect of zinc oxide nanoparticles in Staphylococcus aureus. International Journal of Pharmacy and Biological Sciences, 2 (4): 326-335.

Zhou, H., Fan, T. and Zhang, D. (2007). Hydrothermal synthesis of $\mathrm{ZnO}$ hollow spheres using spherobacterium as biotemplates. Microporous and Mesoporous Materials, 100: 322-327. 